\title{
Pyramidal micromirrors for microsystems and atom chips
}

\author{
M. Trupke, ${ }^{\text {a) }}$ F. Ramirez-Martinez, E. A. Curtis, J. P. Ashmore, S. Eriksson, and E. A. Hinds \\ Blackett Laboratory, Imperial College, Prince Consort Road, London SW7 2BW, United Kingdom
}

Z. Moktadir, C. Gollasch, and M. Kraft

School of Electronics and Computer Science, University of Southampton, Southampton, SO17 1BJ, United Kingdom

\author{
G. Vijaya Prakash ${ }^{\text {b) }}$ and J. J. Baumberg \\ School of Physics and Astronomy, University of Southampton, Southampton, SO17 1BJ, United Kingdom
}

(Received 30 August 2005; accepted 22 December 2005; published online 15 February 2006)

\begin{abstract}
Concave pyramids are created in the (100) surface of a silicon wafer by anisotropic etching in potassium hydroxide. High quality micromirrors are then formed by sputtering gold onto the smooth silicon (111) faces of the pyramids. These mirrors show great promise as high quality optical devices suitable for integration into micro-optoelectromechanical systems and atom chips. We have shown that structures of this shape can be used to laser-cool and hold atoms in a magneto-optical trap.

(C) 2006 American Institute of Physics. [DOI: 10.1063/1.2172412]
\end{abstract}

The miniaturization of optical components leads to higher packaging density and increased speed of devices that manipulate light. This is part of the vast field of Microsystems technology, designated by micro-optoelectromechanical systems (MOEMS), in which electronic, mechanical, and optical devices are integrated on the micron scale. As mirrors are fundamental components of most optical systems, techniques for the integration of high-quality mirrors are relevant for the advancement of this field. In the context of atomic physics, there has been a recent drive to integrate optical elements with atom chips ${ }^{1-3}$ for the purposes of detection and quantum-coherent manipulation of cold atoms. ${ }^{4,5}$ Just as pyramidal mirrors have been used $^{6}$ to form macroscopic magneto-optical traps (MOTs), so these microscopic pyramids may be used to cool and trap an array of small atom clouds on a chip.

We have fabricated two-dimensional arrays of micromirrors in silicon using a method that is simple, economical, and compatible with MOEMS. We start with a (100)-oriented silicon wafer, coated with a thin layer of oxide. Optical lithography is then used to make square openings in the oxide, through which the silicon can be etched. We use the anisotropic etchant potassium hydroxide at a concentration of $25 \%$ by volume and a temperature of $80{ }^{\circ} \mathrm{C}$. This attacks the $\mathrm{Si}(100)$ plane more rapidly than the $\mathrm{Si}(111)$ plane, resulting in a pyramidal pit $^{7}$ bounded by the four surfaces $(1,1,1)$, $(\overline{1}, 1,1),(1, \overline{1}, 1)$, and $(\overline{1}, \overline{1}, 1)$. Typical resulting pyramids are shown in Fig. 1. The $\mathrm{Si}(111)$ faces of the pyramids are expected to be extremely smooth because of the layer-bylayer etching mechanism involved. ${ }^{8,9}$ Atomic force microscope measurements confirm this, giving an rms surface roughness value of less than $0.5 \mathrm{~nm}$ for the uncoated pyramid faces. This makes them ideal as substrates for highquality optical mirrors. After stripping the oxide mask away, a layer of gold of 100 nanometers thickness is applied to the silicon. Gold was chosen as it is a good reflector for infrared light, but other metals or dielectric coatings can also be applied. After sputtering gold, the surface roughness increases

\footnotetext{
${ }^{a)}$ Electronic mail: michael.trupke@imperial.ac.uk

${ }^{b}$ Present address: Department of Physics, IIT, Delhi, India.
}

to $3 \mathrm{~nm}$ (rms). With this amount of roughness one can calculate that the scattering loss of the specularly reflected intensity should be less than $0.5 \%$ in the near-infrared range. ${ }^{10}$

Figure 1(a) shows a small section of the array viewed under a scanning electron microscope after completion of the gold coating. In this particular sample, the square pyramids have $30 \mu \mathrm{m}$ sides and are arranged in a square lattice with a pitch of $100 \mu \mathrm{m}$. Both the etching and the sputtering are standard processes that can be accurately controlled to give reproducible results and to make large numbers of mirrors in a single batch. In the rest of this letter we analyze and measure directly how the pyramids respond to polarized and unpolarized light. We also test a macroscopic model to show that this silicon pyramid mirror geometry is suitable for making a MOT.

The sides of the pyramids define $x$ and $y$ axes, as shown in Fig. 1. Our first test of the mirrors is to illuminate them with a collimated 1-mm-diameter laser beam (wavelength $633 \mathrm{~nm}$ ) propagating along the $z$ axis, i.e., normal to the silicon surface and along the symmetry axis of the pyramids. Figure 2(a) shows the reflected pattern of light observed on a screen $7 \mathrm{~cm}$ away from the mirrors. On this image we have drawn circles indicating the position of spots as expected from a perfect pyramid. The three prominent spots at the corners of the square are due to doubly reflected rays, which (a)

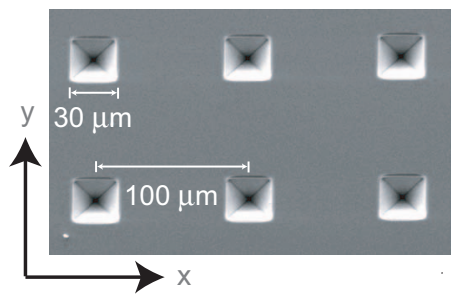

(b)

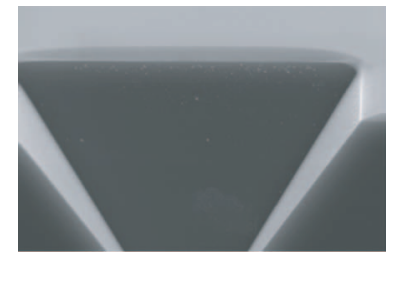

FIG. 1. Scanning electron microscopy micrographs of the etched and goldcoated pyramids. (a) Top view showing pyramids in a rectangular array with a pitch of $100 \mu \mathrm{m}$. (b) Cross-sectional view of a single pyramid. This was obtained by cleaving the pyramid parallel to one of its edges. The base of the pyramid has a side of length $30 \mu \mathrm{m}$, corresponding to a perpendicular depth of $21.3 \mu \mathrm{m}$. 


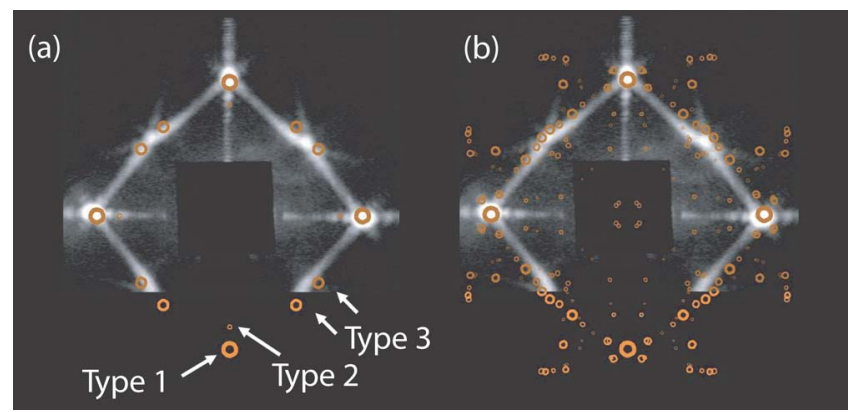

FIG. 2. (Color online) Measured intensity distribution of reflected light, at a distance of $7 \mathrm{~cm}$ from the array of pyramids, when it is illuminated at normal incidence. A central bright spot, which is caused by reflection from the region between pyramids, was blocked to improve the visibility of light reflected from the pyramids. The circles in (a) show the reflection pattern expected for a perfect pyramid, while the circles in (b) indicate the calculated reflection pattern for a pyramid with rounded corners. Size indicates expected relative intensity.

we classify as type (1). These reflect from opposite faces of the pyramid, as illustrated by the solid line in Fig. 3(a). There should be a fourth spot at the bottom of the photographs, but this is blocked by a mount holding the beamsplitter through which the array is illuminated.

If the angle between opposite mirrors is $\alpha$, the type (1) beams make an angle of $(\pi-2 \alpha)$ with the $z$ axis. From the angles measured, we find that $\alpha=(70.6 \pm 0.7)^{\circ}$, in agreement with the expected angle between opposing faces of $\arccos (1 / 3)=70.5^{\circ}$.

When the incident ray is close to the apex of the pyramid (within $1.6 \mu \mathrm{m}$ for a pyramid of $30 \mu \mathrm{m}$ base length), it is reflected twice by the first mirror, as illustrated by the dashed line in Fig. 3(a). These rays, which we call type (2), should produce secondary spots just inside the type (1) spots. However, the power in the type (2) reflected beams is expected to be 100 times smaller because of the small area from which they originate, as shown in Fig. 3(b). Consequently, it is not possible to identify the type (2) beams clearly against the diffracted wings of the type (1) beams. Furthermore, there is a background of light along the $x$ and $y$ axes caused by reflection from rounded edges on the entrance aperture of the pyramid, which can be seen in Fig. 1(b).

If a ray is incident near one of the corners of the pyramid, the first reflection sends it off towards the opposite mirror, but it is intercepted and deflected by the adjacent mirror before the opposite mirror sends it out of the pyramid as a type (3) ray. These rays make an angle of $31.5^{\circ}$ with the $z$

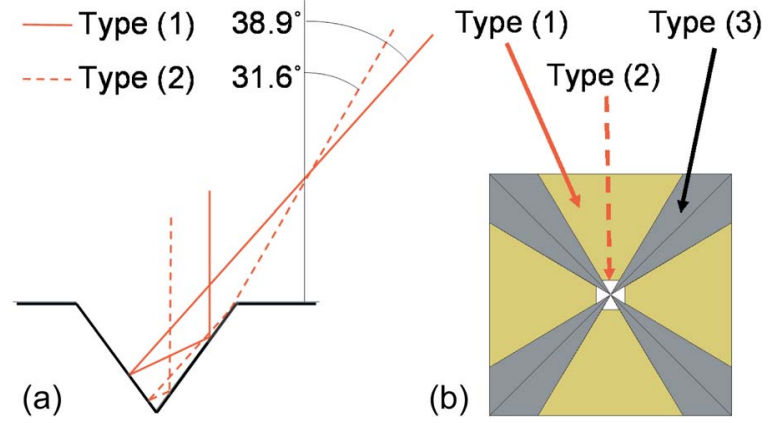

FIG. 3. (Color online) (a) Cross section in the $x-z$ plane through a pyramid, showing type (1) and (2) trajectories. These involve reflections from mirrors on opposite sides of the pyramid. (b) View of the entrance aperture of the pyramid, showing the regions that produce type (1), (2), and (3) rays.

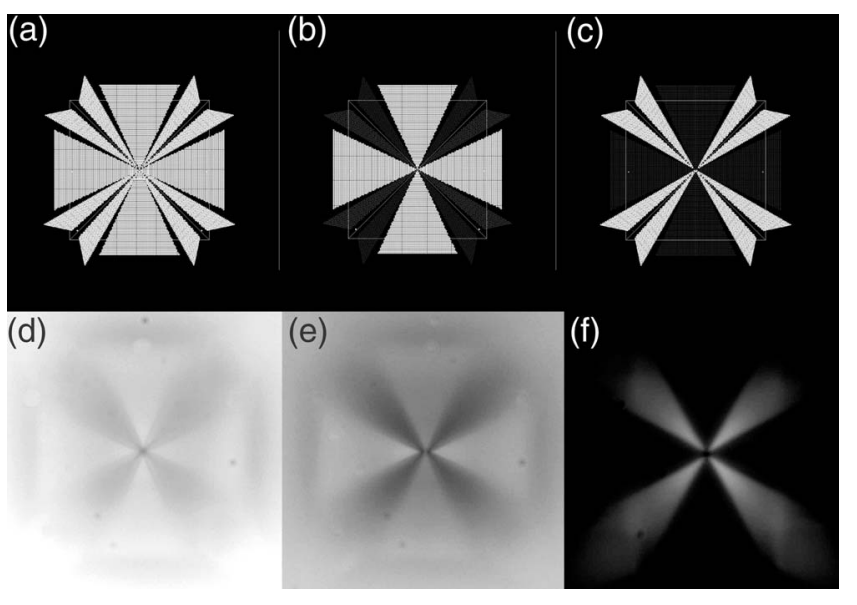

FIG. 4. Views of the vertex pyramidal mirror under an optical microscope at $100 \times$ magnification. Top row: Raytracing simulation. Bottom row: Photographs. (a) and (d) without polarizers; (b) and (e) parallel polarizer and analyzer; (c) and (f) crossed polarizer and analyzer.

axis and form double spots at azimuthal angles of $36.9^{\circ}$, 53. ${ }^{\circ}$, etc. as shown in Fig. 2(a). These spots are less distinct than those of type (1) because the corners of the pyramid are rounded, a feature that does not affect the type (1) rays. Figure 2(b) shows the same photographed reflection pattern, but here the superimposed circles indicate the expected position and magnitude of spots reflected from a pyramid with rounded corners. The roundness is included in the ray-tracing model by four additional surfaces at each corner. These are shaped to form approximate cone sections with radii of $2.5 \mu \mathrm{m}$ at the base and $0.825 \mu \mathrm{m}$ at the apex of the pyramid. The reflection pattern predicted for this modified shape matches many of the features of the photographed intensity distribution more closely.

The three types of ray described earlier also present different characteristics when observed using polarized light. For our observations, we use light which is polarized parallel to the $x$ axis. Reflections of types (1) and (2) leave the linear polarization of the light unchanged in the $x-y$ plane. The type (3) reflections produce rotations of $\pm 53^{\circ}$ or $\pm 78^{\circ}$ about the $z$ axis, depending on whether the pyramid face on which the first bounce occurs is parallel to the polarization of incoming beam. The rotations are caused by the obliquity of the second and third bounces of the type (3) reflections. ${ }^{11}$ This is investigated in our second test of the mirrors, in which we examine them with white light under an optical microscope, illuminating them once again along the $z$ axis. Figure 4(a) shows the image calculated by ray tracing for unpolarized light with the microscope focussed in the plane of the apex of a perfect pyramid. In this figure most of the area is bright. In Fig. 4(b) we show the expected image for linearly polarized light, viewed through a parallel analyser, which suppresses the type (3) contribution. This leads to a reduction in the intensity of reflections from the corner region. In Fig. 4(c), the analyzer is crossed with the polarizer and only type (3) rays contribute, making the corner region bright. The intensity patterns observed in the laboratory are shown in Figs. 4(d)-4(f). They correspond closely to the calculated distributions, indicating that the pyramid reflects light as expected.

Our immediate application for these structures is to build an array of small magneto-optical traps, integrated into an atom chip in a single additional etching step. As in a $90^{\circ}$ pyramid MOT, ${ }^{6}$ lateral confinement is given by the first reAlP license or copyright, see http://apl.aip.org/apl/copyright.jsp 


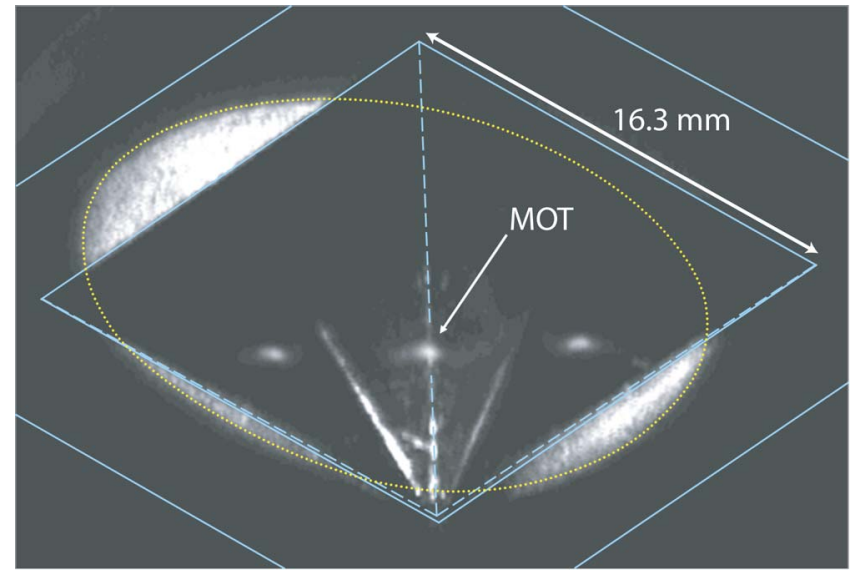

FIG. 5. (Color online). Fluorescence image of $1.6 \times 10^{8}$ atoms magnetooptically trapped in a $70.5^{\circ}$ pyramid. Two reflections are also visible. The $16.3 \mathrm{~mm}$ aperture of the pyramid (solid line) and its sloping edges (dashed lines) have been added to guide the eye of the reader. The outline of the incident laser beam is shown dotted. Some light is scattered from the edges of the pyramid and from the plane outside.

flections of the type (1) beams, while the vertical trapping forces arise from the input beam and the second reflections of the type (1) beams. In the present pyramid the beams are not orthogonal and there are additional rays, which could also disturb the balance of forces in the trap. To test whether it is nonetheless possible to trap atoms in such a pyramid, we constructed a macroscopic glass model with a base length of $16.3 \mathrm{~mm}$, coated with aluminium and a protective layer of $\mathrm{SiO}_{2}$. Figure 5 shows the fluorescence image from a cloud of atoms trapped in this pyramid MOT, together with two reflections of the cloud. There are also reflections from the top face of the pyramid and from the edges. In order to assist the eye, we superimpose the entrance aperture of the pyramid as a solid line and we show the edges dashed. We have also built and tested a $90^{\circ}$ pyramid MOT of similar volume and with the same coating, and find that there is no significant difference in the number of trapped atoms or in the stability of the MOT.

In the microscopic version, we anticipate using pyramids with a $200 \mu \mathrm{m}$ base and will supply the required magnetic quadrupole field using existing microfabrication methods ${ }^{1,12}$ to produce small current loops around each pyramid. We estimate that such a MOT can collect as many as 1000 atoms or as few as 1 , according to the choice of operating parameters. Compared with other methods of creating arrays of microscopic traps on a chip, ${ }^{13,14}$ this relies on a simple fabrication method and requires only a single input laser beam to give all the necessary trapping beams. It has been shown that Bose-Einstein condensation can be achieved on atom chips, both with current-carrying wire traps ${ }^{15}$ and with permanent magnet traps. ${ }^{16}$ Consequently it may be possible to create an array of condensates loaded from these MOTs. Alternatively, if there is just one atom per site, the array would have possible applications in quantum information processing. ${ }^{4}$

Further potential applications for the pyramids are in the areas of photonics and telecommunications. For example, by filling the pits with ferroelectric material or liquid crystals and applying an electric field, it may be possible to use the pyramids as fast optical switches.

In summary, we have designed, fabricated and characterized a new type of micromirror, produced by anisotropic etching through square apertures on a silicon single crystal. As an elementary component for optics, the micromirror has a variety of possible applications in MOEMS devices. We have demonstrated that it is possible to form a magnetooptical trap with this mirror geometry, making these pyramids very promising for creating arrays of microscopic traps on atom chips. Detailed experiments and further theoretical analysis are currently under way to develop these applications.

The authors acknowledge support from the UK EPSRC Basic Technology, QIPIRC, and Physics programmes and from the FASTNET and Atom Chips networks of the European Commission.

${ }^{1}$ R. Folman, P. Krueger, J. Schmiedmayer, J. Denschlag, and C. Henkel, Adv. At. Mol. Phys. 48, 263 (2002).

${ }^{2}$ S. Eriksson, M. Trupke, H. F. Powell, D. Sahagun, C. D. J. Sinclair, E. A. Curtis, B. E. Sauer, E. A. Hinds, Z. Moktadir, C. O. Gollasch, and M. Kraft, Eur. Phys. J. D 35, 135 (2005).

${ }^{3}$ M. Trupke, E. A. Hinds, S. Eriksson, E. A. Curtis, Z. M. E. Kukarenka, and M. Kraft, Appl. Phys. Lett. 87, 211106 (2005).

${ }^{4}$ S. Scheel, J. Pachos, and P. L. Hinds, and E. A. Knight, Quantum Coherence, Lecture Notes in Physics Vol. 689 (Springer, Berlin, 2006), pp. 4780.

${ }^{5}$ P. Horak, B. Klappauf, A. Haase, R. Folman, J. Schmiedmayer, P. Domokos, and E. A. Hinds, Phys. Rev. A 67, 043806 (2003).

${ }^{6}$ K. Lee, J. Kim, H. Noh, and W. Jhe, Opt. Lett. 21, 1177 (1996).

${ }^{7}$ R. Brendel, in Proceedings of the 14th European Photovoltaic Solar Energy Conference, edited by H. A. Ossenbrink, P. Helm, and H. Ehmann (Stephens, Bedford, 1997), p. 1354.

${ }^{8}$ Z. Moktadir and H. Camon, Modell. Simul. Mater. Sci. Eng. 5, 481 (1997).

${ }^{9}$ K. Sato, M. Shikida, T. Yamashiro, M. Tsunekawa, and S. Ito, Sens. Actuators, A A73, 122 (1999).

${ }^{10} \mathrm{P}$. Beckmann and A. Spizzichino, The Scattering of Electromagnetic Waves from Rough Surfaces (Pergamon, New York, 1963).

${ }^{11}$ B. A. E. Saleh and M. C. Teich, Fundamentals of Photonics (Wiley, New York, 1991).

${ }^{12}$ S. Eriksson, F. Ramirez-Martinez, E. Curtis, B. Sauer, P. Nutter, E. Hill, and E. Hinds, Appl. Phys. B B79, 811 (2004).

${ }^{13}$ A. Grabowski and T. Pfau, Eur. Phys. J. D 22, 347 (2003).

${ }^{14}$ E. Hinds, in Atomic Physics 17, AIP Conference Proceedings Vol. 551 edited by E. Arimondo, P. D. Natale, and M. Inguscio (AIP, New York, 2001), pp. 405-413.

${ }^{15}$ H. Ott, J. Fortagh, G. Schlotterbeck, A. Grossmann, and C. Zimmermann, Phys. Rev. Lett. 87, 230401 (2001).

${ }^{16}$ C. D. J. Sinclair, E. A. Curtis, I. Llorente-Garcia, J. A. Retter, B. V. Hall, S. Eriksson, B. E. Sauer, and E. A. Hinds, Phys. Rev. A 72, 031603 (2005). 